\title{
The Model of Mobile Knowledge Services System in the Management of Complex Cluster Project
}

\section{Liu Yong, Liu Bingxin}

School of Business Administration, South China University of Technology Guangzhou, Guangzhou, China Email: liuyong@scut.edu.cn, celinaliu@163.com

Received August 19 ${ }^{\text {th }}, 2010$; revised October 25 ${ }^{\text {th }}, 2010$; accepted November $3^{\text {rd }}, 2010$.

\begin{abstract}
Cluster projects are projects of large-scale or with huge investment, such as Beijing 2008 Olympic Games and Shanghai 2010 Expo. A lot of on-site management works are involved in the process of cluster project management, especially the treatment of sudden accidents which would produce plenty of knowledge and experience. As cluster project on-site management spots change frequently, knowledge produced by mobile workers in the process of on-site management is hard to be saved into the knowledge repository on time. It may cause a knowledge distortion or even missing. On the other hand, in the process of cluster project management, a lot of knowledge is required by on-site workers to deal with on-site activities. However, due to lack of effective mechanisms for knowledge communion, they often face the risk of making wrong decisions. To solve the problems of knowledge management and acquisition in the process of cluster project on-site management, this paper presents a system model for mobile knowledge service.
\end{abstract}

Keywords: Cluster Project Management, Mobile Knowledge Services, Knowledge Service Model

\section{Introduction}

Cluster projects are projects of large-scale or with huge investment, such as Beijing 2008 Olympic Games and Shanghai 2010 Expo [1]. In order to manage these complex cluster projects, large amounts of on-site managers who we call mobile workers are requires. On one hand, in the process of project implementation, a lot of knowledge would be needed as a support and a lot of experience and knowledge are produced as well. If lacking of effective mechanisms for information access and knowledge communion, there would be a risk of making wrong decisions. On the other hand, various experience and knowledge are held by on-site mobile workers [2], but it is difficult for them to record the information and experience produced in the business process into the enterprise's database or repository in real-time. It may result in a distortion of enterprise's information and knowledge, or even missing [3]. The mobile knowledge service system not only can provide the required knowledge for cluster project on-site workers at anytime and anyplace, but also can save the experience and knowledge produced in the process of cluster project on-site management effectively. As a result, the efficiency

supported by" the Fundamental Research Funds for the Central Universities, SCUT ”, project number: 2009ZM0115. of on-site management and the accuracy of decisionmaking would be greatly improved.

At present, there are not systemic researches on the mobile knowledge services for cluster project management at home or abroad. Some related researches are focused on the mobile services of library, information field, mobile communications, mobile learning, and knowledge services. These researches do not connect knowledge service with mobile environment. Generally speaking, mobile knowledge service is a process of providing knowledge service to knowledge users in mobile environment. It can supply individual knowledge services to users at anytime and anyplace by using mobile equipments.

Knowledge produced in the process of project implementation effectively and providing knowledge required by mobile workers accurately have an important practical significance to carry out and manage cluster projects. On one hand, the method to deal with an issue of one project might be also applied to another project or even another cluster project that face the same problem. However, each subproject often works independently and the communion between each other is very little. The reference of the experience and knowledge between different clusters projects is even less. So it resulted in the 
repetition of similar problems and a serious waste of resources. On the other hand, if these experience and knowledge could be collected and used by all the subprojects' workers through a simple and convenient way, the efficiency of implementing complex clusters would be greatly improved.

In order to help mobile workers to record experience and knowledge produced in the process of project implementation as well as to acquire the knowledge that they need in mobile environment; this paper presents a system model based on mobile knowledge services.

\section{Design of Mobile Knowledge Services System}

\subsection{The Description of the System Model}

To supply the required services to mobile workers of complex cluster projects at anytime and anywhere, mobile terminals should be chosen firstly. Mobile terminals such as mobile phone, PDA, notebook computers are commonly used now. However, the volume of notebook computers is large and not very convenient to be carried-on. Mobile phone also has some disadvantages such as small memory, slow rate of data transmission, small screen which caused inconvenient operation. Compared with them, PDA (Personal Digital Assistant) has been widely used in systems based on mobile services because it is not only easy to be carried and operated, but also has advantages of good display of LCD screen and the capability of wireless communication [4]. As the operating system of PDA is designed to face users directly and is similar to traditional computer operation, so it can be easily mastered by mobile workers. At the same time, PDA can provide various wireless technologies and we can go through internet and get the required information through the built-in Modem or optional wireless inter- net groupware. So, we choose PDA as our mobile terminal.

To achieve the real-time linkage between client and system in mobile environment, the appropriate wireless access technology is also needed besides mobile equipments. There are two ways of wireless access that can be used by Internet mobile users: dial-up or through a wireless network card to log on to wireless private network. Although a higher data transfer rate could be achieved by the way of dial-up, it would be enslaved by environment and not convenient to be used by mobile workers at anytime and anyplace. Wireless Private Network is the mainstream in the future, with which we can easily access the Internet anytime. At present, the popular wireless access technologies include GSM, CDMA, GPRS, CDPD and LMDS. Compared with other technologies, CDPD access technology which takes a method of packet data is an open system architecture based on TCP/IP. It combines the functions of open interface, high transmission speed, user unit determination, air link encryption, air data encryption, and compressed data error correction, and wireless access by using the world standard IP address mode. It also provides the seamless connectivity of the same network layer and multi-protocol network services. So it is widely recognized as the best wireless public network data communication protocol [5]. Therefore, we choose CDPD access technology to achieve wireless access of mobile equipments in this paper.

With mobile equipments and access technology, the probability of using mobile knowledge services system is assured in theory. In the following, we define a system model of mobile knowledge services used in the process of complex cluster project management. Figure $\mathbf{1}$ is a schematic figure of the model structure.

From Figure 1 we can see that the system is divided into three levels: user layer, application layer of mobile

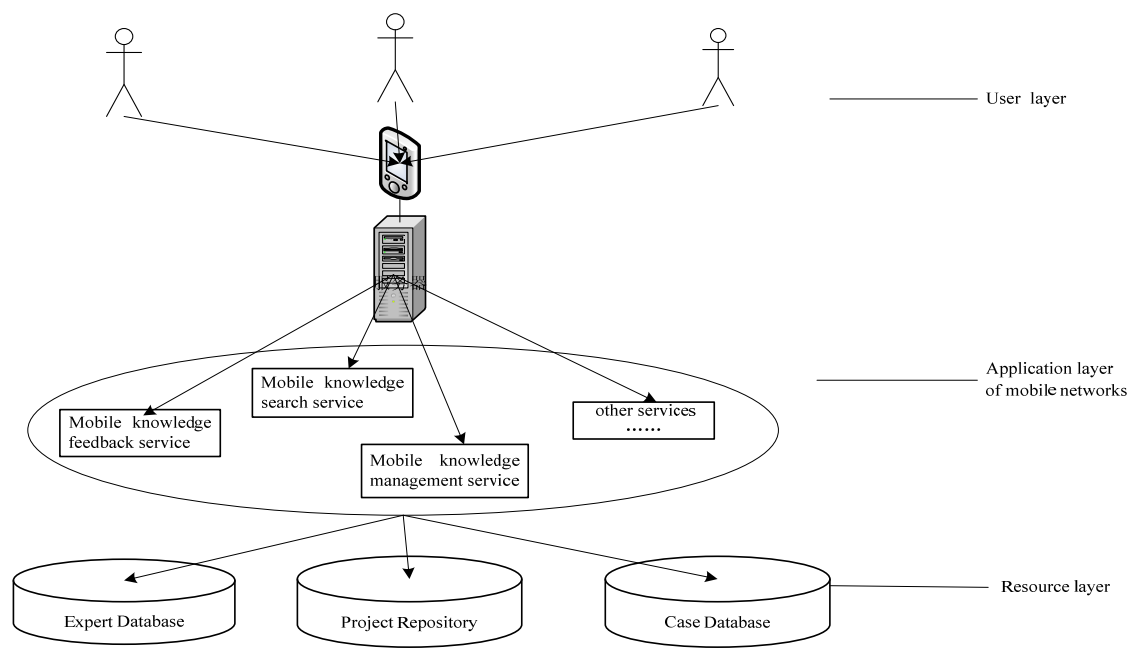

Figure 1. The system model structure of mobile knowledge services in complex cluster project. 
networks and resource layer. The on-site workers of complex cluster project, who are users of the system, can use PDA as mobile equipments to select services that they need. The mobile network application layer is mainly used for receiving users' request and sending the information that they need. The request sponsored by the client program is sent out after business is selected through the built-in browser of a mobile terminal [6]. All the information needed in the process of system operating are provided by the resource layer including knowledge repository, experts database and case database of the project and the relevant information uploaded by users are also stored in the corresponding database.

\subsection{The Function Model of Mobile Knowledge Services System}

Mobile Knowledge services of complex cluster project can be mainly divided into mobile knowledge management and mobile knowledge acquisition. The former service pattern mainly accomplishes the function of collecting and saving project management knowledge. The latter pattern mainly accomplishes the function of providing knowledge to required workers through the ways of information search, knowledge quiz, hot information feedback, WAP access, information discussion platform, tracking knowledge services.

1) Mobile Knowledge Management:

Each subproject of complex cluster project can be regarded as a separate project, including feasibility study, project establishment, project planning, project implementation, acceptance and check items etc. Mobile workers can use PDA to login the knowledge services system of cluster project, and record problems they meet with.

Most of those who need to use the mobile knowledge services system to upload experience data are on-site management workers at the stage of project implementation. Therefore, we divide the problems happened in project implementation phase into two categories: daily management and emergency. On-site management workers use event data sheet which is set in the system already to record the problems they meet with and the solutions of problems after choosing the respective categories. Then, they can save the contents and upload them to the system. The event data sheet is shown in Table $\mathbf{1 .}$ Through the record of each event, the problems occurred in the implementation phase of each subproject and the solutions can be saved effectively. On one hand, it can help their team members and leaders know the general situation of the subproject better. On the other hand, it is also convenient for participants of other subprojects to realize and learn from them.

Figure 2 shows that how on-site workers of cluster
Table 1. Event data sheet.

\begin{tabular}{cc}
\hline Name of Event & \\
\hline Time & Spot \\
Description & \\
Solution and & \\
Treatment Scheme & \\
Effect Evaluation & Reference to \\
Scheme Presenter & Familiar Project \\
\hline Reporter: & Participator \\
\hline
\end{tabular}

project use PDA and mobile knowledge services system to record events.

It indicates that worker 1,2,3,4 etc. of each subproject use mobile knowledge services system to upload problems and solutions happened in the implementation phase of project through PDA. They save the information under respective categories.

2) Mobile Knowledge Acquisition

Mobile knowledge acquisition services can be divided into ordinary knowledge services pattern and tracking knowledge services pattern. Ordinary knowledge services pattern still can be divided into five ways: information search, knowledge quiz, hot information feedback, WAP access, and information discussion platform. Ordinary knowledge services pattern mainly deals with clear or vague demanded information from mobile workers who have a simple understanding and a strong purpose about what type of services they need before choosing this service. Tracking mobile knowledge services pattern asks knowledge providers to supply follow-up services. It not only can deal with mobile workers' clean or vague demanded information, but also supply guidelines for potential demand.

a) Information Search

All workers of the cluster project can search for information that they need in the way of inputting keywords after logging-in system. The system can provide all relevant information that mobile workers have uploaded to the system as well as knowledge that experts have collected in repository. It may include documents, pictures, videos and other formats. Through this way, all workers of cluster project are available to realize the process of each subproject and encountered problems conveniently. When dealing with a problem, they can check whether other subprojects have occurred it or not and their solutions. Through this method, the problem of resources waste which is cause by little communication between various subprojects can be mitigated rapidly and work efficiency can be improved. On the other hand, mobile workers can get professional knowledge existed in the project database according to their needs. It can satisfy mobile workers' demand for knowledge at anytime and anyplace. System would also provide a feed- 


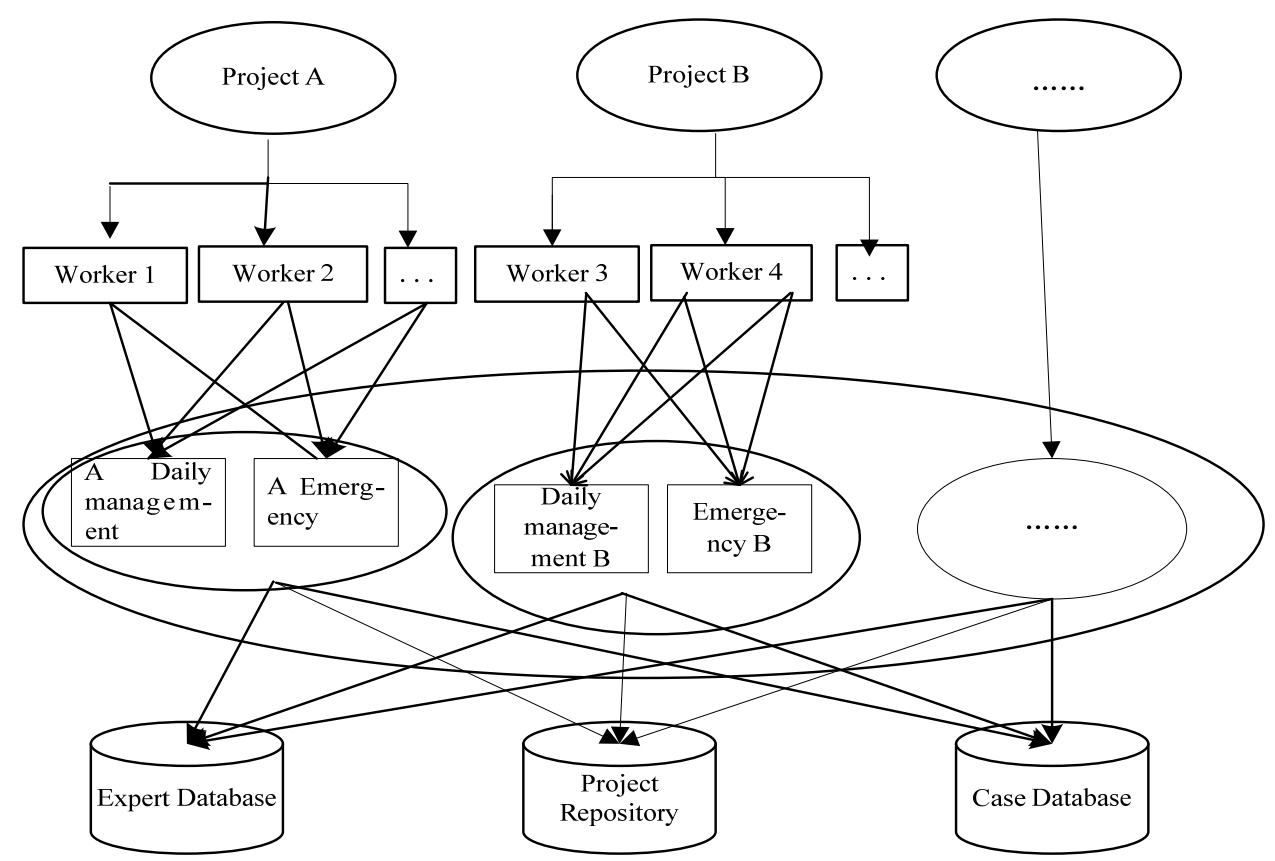

Figure 2. The sketch map of uploading events by mobile workers by using system.

back function, if a worker is unable to find the corresponding knowledge, he can use the feedback function to submit the issue to system. System maintenance workers would carry out these problems regularly and put new solutions into the project database.

b) Knowledge Quiz

If mobile workers don't have enough time to search for information by themselves, they can use the function of knowledge quiz. They only need to input the information about their requirement into problem table setting in the system. The system will return the matching answers to users automatically. As the multimedia mail system can achieve the interoperability with Internet and provide the function of sending messages between mobile terminals and Internet [7], therefore, after the completed problem table is sent to the mobile knowledge service system, the answer will return to the mobile worker's PDA in the form of multimedia mail through background processing. The contents of the mail can include text, sound, picture, and video and so on. Using multimedia mail, we can transmit plentiful information to ensure the quality of the solution. If a mobile worker's requirement cannot be matched in project database, the system will automatically reply no matching knowledge to him. At the same time, the problem table will be stored in a particular position waiting for expert to solve it. After solving the problems, the results would be saved in the database. So, the database can be enriched continuously.

c) Hot Information Feedback

System can regularly send the latest information that mobile workers concern in the form of multimedia mails by recording demand information that each mobile worker often send. So the mobile workers could see the latest developments in their concerned fields without searching by themselves. In this way, it can reduce the time to search information and avoid the geographic or tool's limited. This kind of service is called hot information feedback.

d) WAP Access

Through classifying the knowledge, mobile knowledge services system maintenance workers show them out in the form of ordinary website and put related links to the web page in order to achieve resource sharing. Mobile workers use PDA to login the system, then they can visit the website and download the data that they need. All information about cluster project will be released on the web timely. As a result, mobile workers can realize the progress of the project at anytime and anyplace. Through arranging data and analyzing browsed information regularly, website maintenance workers would expand data which are often browsed and downloaded to update the web timely.

e) Information Discussion Platform

The mobile information discussion platform is divided into two types: the platform between mobile workers and the platform between mobile workers and experts. The first one allows mobile workers to discuss with each other. After entering the platform by PDA, they can describe their problems or answer someone else's problems at any moment. Through this way, we 
can ensure the exchange of knowledge and the resource-sharing freely.

Mobile knowledge services system also provides a platform for mobile workers to communicate with experts. They can discuss with experts on this platform at anytime and anyplace. Experts will give their opinions to questioners and mobile workers also can discuss the ultimate solutions according their own actual situation with experts. In the process, experts can transfer mobile workers' tacit knowledge such as experience into the explicit knowledge. Then they put the new explicit knowledge into the database. As a result, the aim of knowledge innovation and value-added is achieved.

f) Tracking Mobile Knowledge Service

The pattern of tracking mobile knowledge services is applicable to the mobile workers who have a lot of requirement or the problems can't be completed after a short time guidance and need long-term service. Through tracking mobile knowledge services, we can know experts' opinions at anytime and anyplace which ensure to finish the work smoothly. After choosing this service, we can choose a knowledge service worker according to the required fields. Then we send our requirement to him/her through multimedia mail. It is a one-to-one communication. Mobile workers also can choose to discuss with experts by information discussion platform timely.

In the whole process of solving problems, knowledge service workers are required to integrate into the decision-making process with mobile workers to establish a harmonious "user - service worker" relationship as featuring lawyer. The service duty system pointing to specific mobile workers should be established, and then they can do integrative services from knowledge collection, disjunction, reorganization, innovation, integration to application [8]. Knowledge service workers not only should integrate into the process of solving problems to solve the mobile workers' existing problems, but also should help them to grasp the whole problems from macroscopic side. Based on the detailed understanding of mobile workers' working environment and requirement, knowledge service workers may realize their potential demand and provide diversiform services.

3) Mobile Knowledge Acquisition

In addition to the existing two mobile services modes of mobile knowledge management and mobile knowledge acquisition, we also can use the statistical results, which extract from the information submitted by the mobile workers to develop two new functions.

a) Reward Management

In order to encourage mobile workers to upload information through system, we can set up a function of reward management within the system. That is, in a certain period of time, the system automatically counts the total number of information uploaded by all mobile workers in the cluster project or that of each subproject and shows these numbers out in the form of statistical tables according to the requirement. The workers who upload a mass of information can be given encouragement in the form of bonuses. The system also shows award out in statistical table. By this way, we can inspire the enthusiasm of mobile workers to save explicit and tacit knowledge produced at the stage of project implementation as many as possible.

b) Statistics Analysis

According to the categories of events uploaded by mobile workers, the system automatically counts and shows the number about some related issues or problems occurring frequently at some stages of the project. It can make leaders realize which problems often occur in the process of project implementation or which problems are easy to happen in a subproject or other questions only through using the system. All the information can intuitively get from statistical data. It is easy to make leaders understand the problems of the whole cluster project completely and the overall progress of the project.

The development of two new functions can further improve the system's efficiency and make the range of system's users expand to project senior managers from normal mobile workers. It not only can make the system collect and provide knowledge, but also can provide decision support for the project decision-makers.

Through the definition of the system and functions above, we can conclude the function diagram of complex cluster project mobile services system. It is shown in Figure 3.

\section{Case Study}

The mode of mobile knowledge services system has been applied into the actual cluster projects. Figure 4 shows how knowledge flow inside one cluster project before mobile knowledge service system is used.

Figure 4 shows the flowing direction of knowledge inside the cluster project. Two-way arrow indicates the strong flow of information and no arrow shows little exchange of information. From the figure we can see two problems. On one hand, the knowledge produced in the process of cluster project implementation, is transferred in this order: on-site workers - responsible officers department managers - project managers - total leader of cluster project. It is a transmission with levels as well as a bidirectional flowing. However, it not only takes a long period of time to transfer information, but also causes information distortion. On the other hand, as each subproject act independently, the communion among different subprojects and different departments are not so much. In the picture above, an ideal condition is that all 


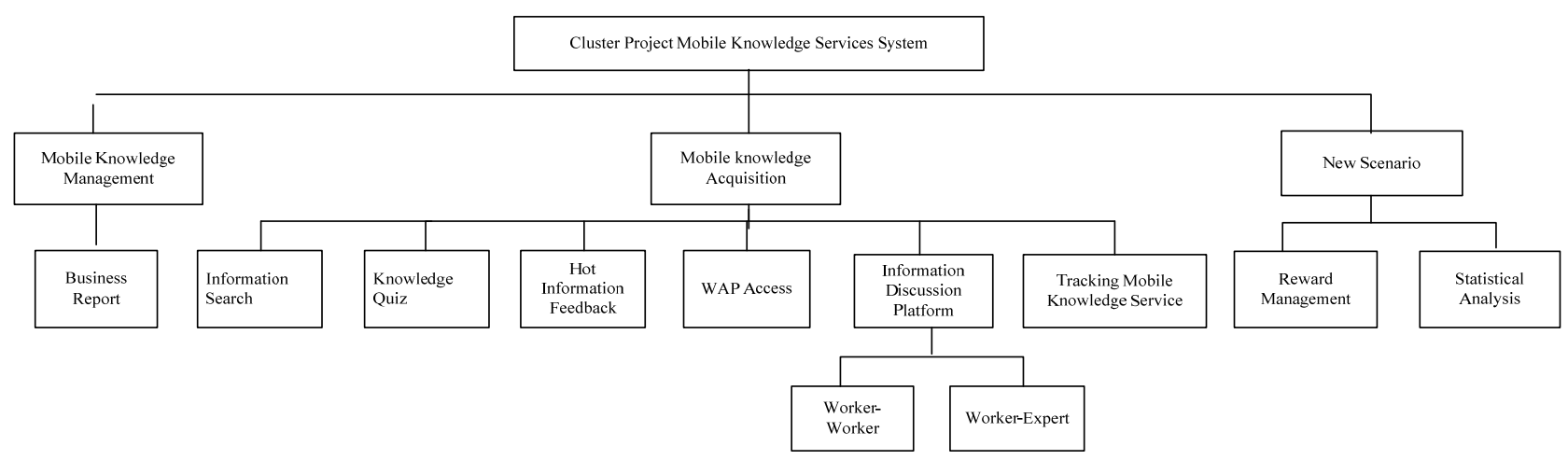

Figure 3. The function diagram of complex cluster project mobile services system.

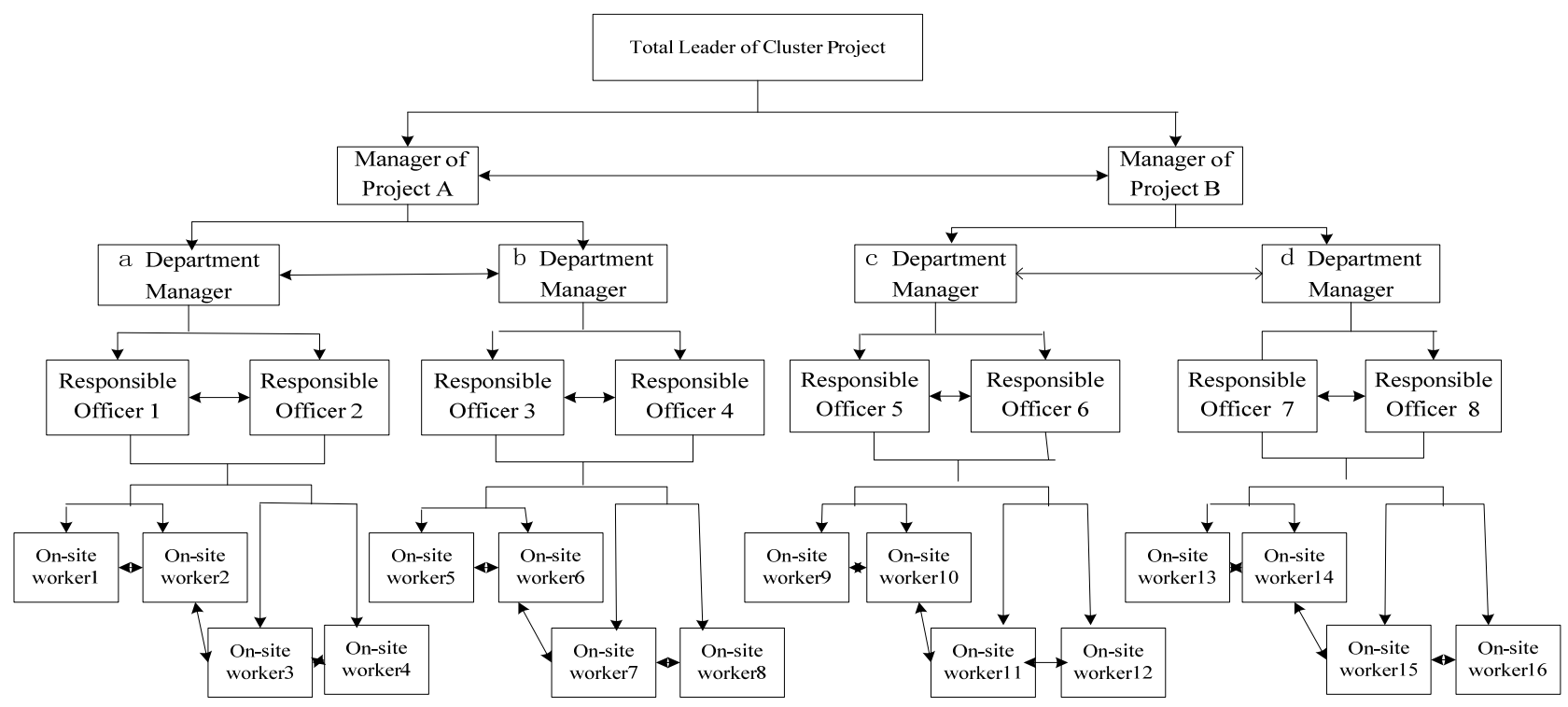

Figure 4. The function diagram of complex cluster project mobile services system.

people should have two-way arrows connection. But in fact, there is little communication among the different subprojects or the same project with different levels. If experience and knowledge generated in the process of cluster project implementation can be used by all participants, the efficiency would be greatly improved and the probability of making mistakes would be reduced.

Using mobile knowledge services system can solve these two problems well. First of all, workers in any level of the project can use the system to get a comprehensive understanding of events that are happened in the process of project implementation. Especially for project managers and total leader, they can know about the situation at work site anytime and anywhere without going there. Secondly, for all on-site mobile workers, knowledge service system can tell them about problems that other people have encountered and their solutions. So they can learn from each other. It is an effective method to solve the problem of communicating inconveniently between mobile workers and the difficulty to learn experience from different cluster projects. Due to the system everybody can make detailed and comprehensive communication without meeting each other.

In order to use PDA to upload events, the first thing that mobile workers should do is to login the system and choose the function of business report. In view of the confidentiality requirement, we cover the important information of the operation system to form the following diagram shown in Figure 5.

Figure 5 shows that after mobile workers select the function of business report, the pull-down menu appears all subprojects' names. If we select subproject B, all stages of project would be showed in the side-menu. Then we can choose the stage of the event belonged, for example, the stage of project implementation. Further we select the categories of the event - daily management. Then we can click the button of "Next" to enter the page of event data sheet. 
Figure 6 is the page for recording event. The upper left corner shows the operation order we have done: Business Report - Project B - implementation. If mobile workers do false operation, he can click the "Quit" button to return to the previous page. If the operation is correct, he can continue to fill the blank. Clicking a space on the blank, the system would enter into a special page for mobile workers to complete the description. After completing a description, click "Finish" to return to Figure 6 and go on filling other blanks. If all blanks are completed, click the button of "Upload" to upload it into the system. Then other workers can view it. If there is an interruption, we can click "Save". So the information is saved in system for a while and would not be uploaded until we complete the fillings in the next time.

Other functions of this system are also applied in the process of cluster project management. It can solve the problems of knowledge management and acquisition of on-site management well. To sum up, it is convenient to provide appropriate knowledge services for on-site mobile workers of the cluster project management team.

\section{Conclusions}

This paper presents a system model that can be applied to the management of complex cluster projects. It is made up of three layers: user layer, application layer of mobile networks and resource layer. In this paper, mobile know-

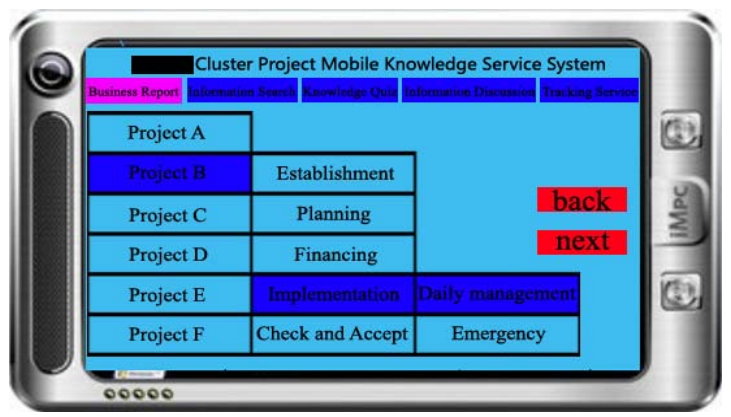

Figure 5. the operation page of choosing function of business report.

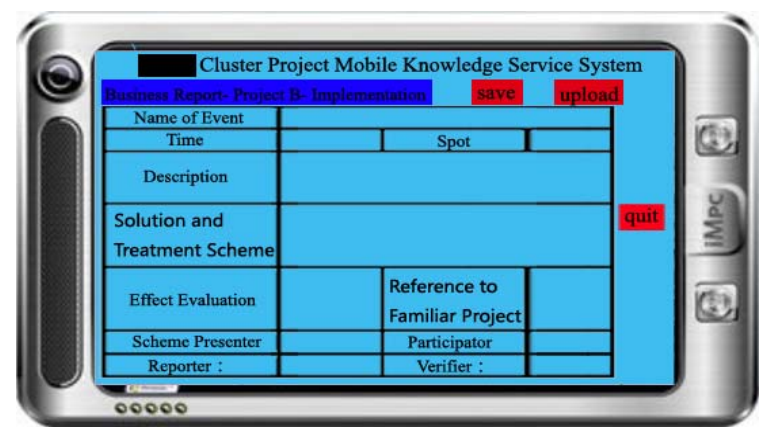

Figure 6. The operation page of event data sheet. ledge services of complex cluster project is summarized into two service modes which include mobile knowledge management and mobile knowledge acquisition. The service of mobile knowledge management is mainly to complete the collection of mobile workers' experience and knowledge. The service of mobile knowledge acquisition comprehensively meet the knowledge requirement of mobile workers through six ways which are information search, knowledge quiz, hot information feedback, WAP access, information discussion platform, and tracking mobile knowledge services. Through the application in the process of complex cluster project management, this system model can meet the demand of knowledge services for mobile workers in the team of complex cluster project management.

\section{REFERENCES}

[1] Y. Cheng and H. Y. Liu, "The PSO Analysis of Large Clusters Project - the Research of Expo Project," Architecture Software, Vol. 2, No. 27, 2005, pp. 53-56.

[2] B. Skattor, "Design of Mobile Services Supporting Knowledge Processes on Building Sites," Management of e-Business, Eighth World Congress , 11-13 July 2007, p. 10. doi:10.1109/WCMEB.2007.6

[3] K. J. Liao and Q. H. Liu, “An Architecture of Mobile Knowledge Service Based on Document Knowledge Base \& Its Application. Management of e-Commerce and e-Government," International Conference, 16-19 September 2009, pp. 404-407.

[4] C. W. Li and D. Li, "A System Model Based on Mobile Service. Network and Communication,” Vol. 26, No. 2-3, 2010, pp. 127-128.

[5] H. G. Cui, "The Research and Implementation on Knowledge Management Technology in Mobile E-Government Systems," Guangdong University of Technology, China, 2002, pp. 12-14.

[6] X. Q. Zhang and F. C. Yang, "A Model of Mobile Business Operation Service Based on the Network," Journal of Beijing University of Posts and Telecommunications, Vol. 29, 2006, pp. 189-193.

[7] Y. X. Yangm and J. X. Wang, "Research on the Mobile Learning Mode Based on 3G Technology," Network Education and Modern Long-distance Education, Vol. 8, 2005, pp. 37-39.

[8] J. Q. Li, "The Research on the Characteristics and Pattern of Knowledge Service,” Information and Documentation Services, Vol. 2, 2004, pp. 16-19.

[9] K. J. Liao, Q. H. Liu and X. L. Zhao, “Ontology-Based Model of Mobile Knowledge Service for the Inspection of Construction Project," Management and Service Science, 2009, MASS '09 International Conference, 20-22 September 2009, pp. 1-5.

[10] T. Yin, "The Education Information Strategy Based on Knowledge Service and Information Management," Management World, Vol. 7, 2006, pp. 153-154 
[11] N. H. Kuang, Q. Y. Hu and R. Du, “A Type of Management Pattern of 'Direct - Services-Prompting' in Knowledge-Based Enterprises,” Journal of Management Sciences in China, 2004.
[12] H. Evanschitzky and D. Ahlert, "Knowledge Management in Knowledge-Intensive Service Networks," Management Decision, Vol. 45, 2006, pp. 265-283. doi:10.1108/00251740710727287 